\title{
The Phosphoinositides: Key Regulators of Salmonella Containing Vacuole (SCV) Trafficking and Identity
}

\author{
M.C. Kerr, N.A. Castro, S. Karunaratne and R.D. Teasdale \\ Institute for Molecular Bioscience, The University of Queensland \\ Australia
}

\section{Introduction}

Derived from the base of membrane ruffles in response to growth factor stimulation (Haigler, McKanna et al., 1979), the macropinosome is a large (diameters $>0.2 \mu \mathrm{m}$ ) phasebright endocytic organelle that is readily labelled with fluid-phase markers. It is the primary means by which macrophages sample their immediate environment for antigens, is essential for proper renal function, is intrinsically linked to cellular migration and has a major role in the down-regulation of signalling from cell surface receptors (Kerr and Teasdale, 2009; Swanson and Watts, 1995). To promote invasion and survival, Salmonella subverts the host cell's normal macropinocytic machinery to gain entry into the nonphagocytic epithelial cells of the intestinal wall. Upon binding to the host cell surface the pathogen utilises a specialised apparatus called the type III secretion system (T3SS) to deliver a suite of bacterial virulence proteins directly into the host cell's cytoplasm. Salmonellae encode two distinct T3SSs within Salmonella Pathogenicity islands 1 and 2 (SPI1 and SPI2) that function at discrete stages of the infection. Whilst SPI1-T3SS is predominantly active on contact with the host cell's surface and serves to translocate virulence proteins across the plasma membrane, driving cytoskeletal rearrangements and signalling events that promote the uptake of the pathogen, SPI2-T3SS is active within intracellular compartments during the later stages of infection to generate a replicative niche (Haraga, Ohlson et al., 2008). As the environment of the encompassing macropinosome, also called the Salmonella Containing Vacuole (SCV) acidifies and matures, losing markers of the early endosomal system like transferrin receptor, EEA1 and Rab5, Salmonellae undergo extensive bacterial surface remodelling and expression and assembly of SPI2-T3SS is induced. The SPI2-T3SS enables the translocation of virulence factors across the SCV membrane into the host cell's cytoplasm. These virulence factors initiate a dramatic alteration in the host cell's vesicular trafficking pathways leading to the accumulation of late endosomal markers like Rab7 and LAMP1 and 2 on the SCV and the formation of long filamentous membrane structures. These Salmonella-induced filaments (SIFs) originate from the SCV, are LAMP1-positive, and function to increase the size of the $\mathrm{SCV}$ in a specific and directional fashion to accommodate bacterial replication during systemic infection as well as provide nutrients to the isolated pathogen (Garcia-del 
Portillo and Finlay, 1995; Garcia-del Portillo, Zwick et al., 1993a, b; Haraga, Ohlson et al., 2008). What is clear from the literature is that this entire process reflects a carefully choreographed interaction between the bacterial virulence factors and the molecular machinery of the host cell.

\section{Phosphoinositides and their effectors, tightly controlled regulators}

Phosphoinositides (PI), the phosphorylated derivatives of the lipid phosphatidylinositol (PtdIns), can be singly or multiply phosphorylated on the $3^{\prime}, 4^{\prime}$, and $5^{\prime}$ position of the inositol headgroup to generate 7 distinct PI isoforms (Vicinanza, D'Angelo et al., 2008). Reversibly phosphorylated in a tightly regulated fashion by phosphatases and kinases that are heterogeneously localised within the cell, the PIs are consequently enriched on the cytosolic face of distinct intracellular membranes (Di Paolo and De Camilli, 2006). For example, the most abundant PIs, $\mathrm{PI}(4,5) \mathrm{P}_{2}$ and $\mathrm{PI}(4) \mathrm{P}$, are each constitutively present in the cytosolic leaflet of the plasma membrane and Golgi apparatus respectively whilst the 3phosphorylated PIs, $\mathrm{PI}(3) \mathrm{P}, \mathrm{PI}(3,4) \mathrm{P}_{2}$ and $\mathrm{PI}(3,5) \mathrm{P}_{2}$ are found distributed throughout the endolysosomal system. The relative amounts of the PIs also vary dramatically between and within cells. Virtually undetectable in quiescent cells, $\mathrm{PI}(3,4,5) \mathrm{P}_{3}$ levels rapidly spike upon stimulation and during specialised membrane trafficking events through the coordinated and regulated activity of class Ia PI3-kinase phosphorylating the $3^{\prime}$ position of $\mathrm{PI}(4,5) \mathrm{P}_{2}$ (Vanhaesebroeck, Leevers et al., 2001).

Remarkably complex, PI metabolism represents a delicate equilibrium balancing the relative abundance and position of these lipids within the cell. Briefly, PtdIns is converted to PI(3)P or PI(4)P on endosomes or the Golgi through the actions of vacuolar protein sorting (Vps) 34-p150 and $\mathrm{PI}(4) \mathrm{KII}<$ respectively. Additionally, conversion of $\mathrm{PI}(3,4,5) \mathrm{P}_{3}$ to $\mathrm{PI}(3) \mathrm{P}$ on nascent endocytic compartments may be the consequence of the sequential dephosphorylation of $\mathrm{PI}(3,4,5) \mathrm{P}_{3}$ as catalysed by 4 - and 5-phosphatases. Src homology 2 domain-containing inositol 5-phosphatase (SHIP) 1 and 2 are potential 5-phosphatase candidates, dephosphorylating $\mathrm{PI}(3,4,5) \mathrm{P}_{3}$ to $\mathrm{PI}(3,4) \mathrm{P}_{2}$ at the cell surface or on newly formed endocytic structures. Type I and II 4-phosphatases may then catalyse the conversion between $\mathrm{PI}(3,4) \mathrm{P}_{2}$ and $\mathrm{PI}(3) \mathrm{P}$ (Krauss and Haucke, 2007). Alternatively $\mathrm{PI}(3,4,5) \mathrm{P}_{3}$ may simply be lost from the endocytic membrane and VPS34-p150, an effector of early endosomal Rab5, could drive the de novo synthesis of PI(3)P from PtdIns (see Figure 1) (Zerial and McBride, 2001).

Relatively high concentrations of $\mathrm{PI}(4,5) \mathrm{P}_{2}$ are constitutively maintained at the plasma membrane primarily through the actions of a diverse family of PI(4)P 5-kinases and the 3phosphatases. In addition to being intrinsic to numerous signalling, cytoskeletal and endocytic events $\mathrm{PI}(4,5) \mathrm{P}_{2}$ also serves as a precursor to $\mathrm{PI}(4) \mathrm{P}$ contributing to the pool found predominantly within the Golgi Apparatus and in secretory granules (Levine and Munro, 2002; Panaretou, Domin et al., 1997; Wang, Wang et al., 2003). The accumulation of PI(4)P within the Golgi reflects the presence of multiple $\mathrm{PI}(4) \mathrm{Ks}$ and $\mathrm{PI}(4,5) \mathrm{P}_{2}$ phosphatases in conjunction with relatively low levels PI(4)P 5-kinase activity.

The most recently identified of the PIs, $\mathrm{PI}(3,5) \mathrm{P}_{2}$, is synthesised from $\mathrm{PI}(3) \mathrm{P}$ by the $\mathrm{PI}(5)$ kinase, PIKfyve (Shisheva, 2008). Whilst the precise role $\mathrm{PI}(3,5) \mathrm{P}_{2}$ plays in the mammalian 

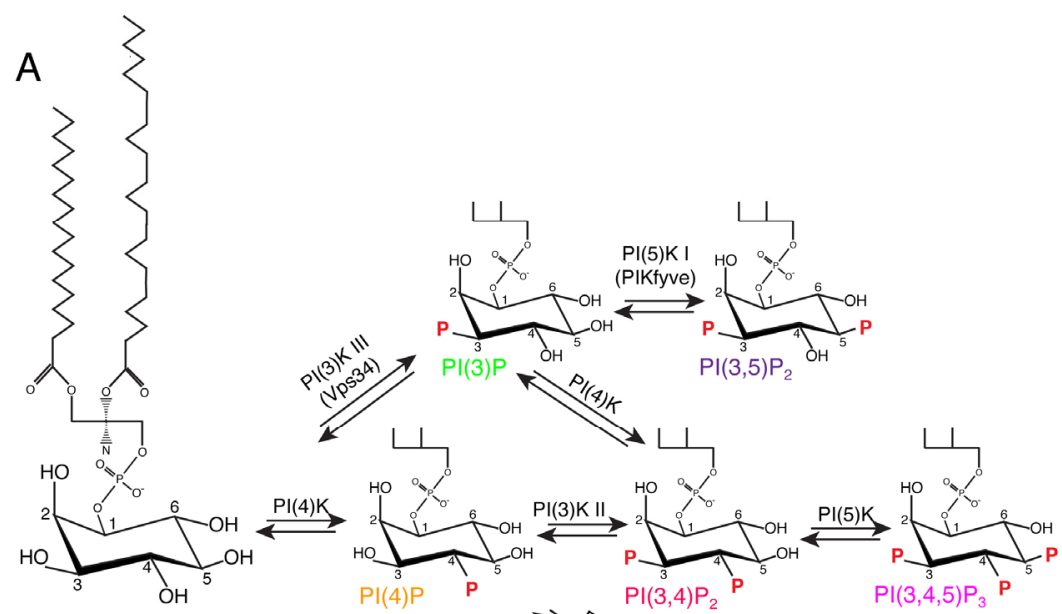

Ptdlns

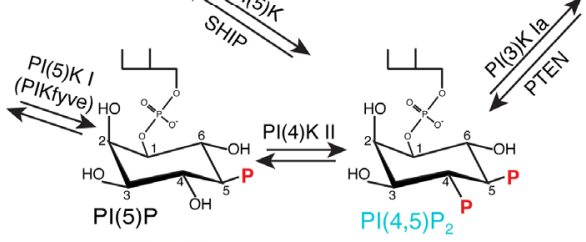

B

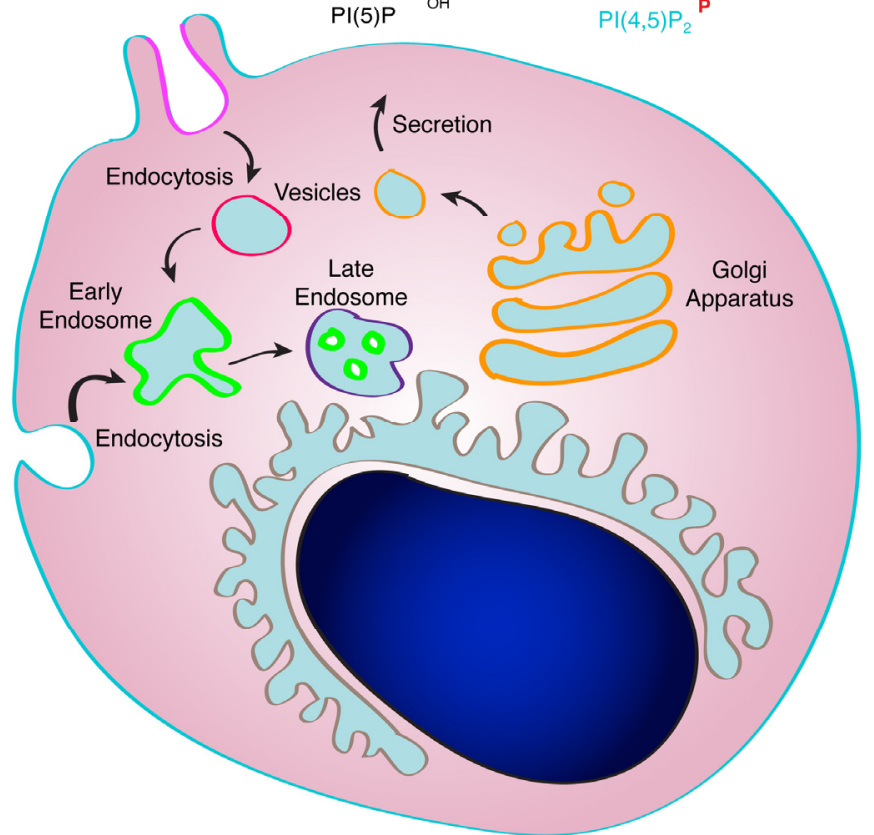

Fig. 1. PI metabolism and subcellular distribution. The phosphoinositides are phosphorylated derivatives of phosphatidylinositol. Their metabolism is regulated by kinases and phosphatases that are distributed heterogenously throughout the cell contributing to the accumulation of specific PIs in discrete intracellular membranes. 
system is currently emergent, disruption of the yeast PIKfyve equivalent, Fab1p, results in a highly complex phenotype. The observation that a $\triangle F A B 1$ strain entirely devoid of $\mathrm{PI}(3,5) \mathrm{P}_{2}$ displays a dramatically enlarged vacuole that fails to acidify, partial defects in prevacuolar compartment (PVC; mammalian endosome equivalent) sorting, defective inheritance of vacuoles in daughter cells and a reduction in the number of intravacuolar vesicles seen by electron microscopy all suggest that $\mathrm{PI}(3,5) \mathrm{P}_{2}$ is integral to a number of processes essential for the maintenance of vacuolar/lysosomal system (Cooke, 2002; Cooke, Dove et al., 1998; Dove, McEwen et al., 1999; Dove, McEwen et al., 2002; Dove, Piper et al., 2004; Gary, Wurmser et al., 1998; Odorizzi, Babst et al., 1998; Yamamoto, DeWald et al., 1995). In agreement, we utilised time-lapse videomicroscopy to demonstrate that PIKfyve activity is essential for the fusion of macropinosomes with late endosomal/lysosomal membranes (Kerr, Wang et al., 2010).

The unique spatial and temporal distribution of the PIs provides the mechanism for the exquisite control with which this protein-lipid network regulates membrane trafficking and signalling events. Specifically, they serve as membrane anchors to recruit a suite of PI-binding molecules of diverse function through a variety of domains with differing PIaffinities and -specificities. The most thoroughly investigated of these, the plekstrin homology $(\mathrm{PH})$ domain, is a $\sim 120$ residue motif found in 275 human proteins (DiNitto and Lambright, 2006; Lemmon and Ferguson, 2000). The relatively low sequence conservation between $\mathrm{PH}$ domains is reflected in the significant variation in specificity and affinity for individual PIs. For example, the PH domains of Grp1 and PLC $\delta 1$ each bind PI $(3,4,5) \mathrm{P}_{3}$ and $\mathrm{PI}(4,5) \mathrm{P}_{2}$ respectively, whilst those of DAPP1, PDK1 and PKB bind both $\mathrm{PI}(3,4) \mathrm{P}_{2}$ and $\mathrm{PI}(3,4,5) \mathrm{P}_{3}$ (Cronin, DiNitto et al., 2004; DiNitto and Lambright, 2006; Lemmon and Ferguson, 2000).

In contrast, the FYVE domain, named after the four cysteine-rich proteins in which it was first identified (Fab1, YOTB, Vac1, and EEA1) after, is a $\sim 70$ residue zinc-binding finger found in 28 human proteins that displays remarkably high affinity and specificity for PI(3)P. It serves to localise proteins with this domain predominantly to PI(3)P-enriched early endosomes, multivesicular bodies, phagosomes and macropinosomes (Cronin, DiNitto et al., 2004; DiNitto and Lambright, 2006; Lemmon and Ferguson, 2000). The afore-mentioned PI(5) kinase, PIKfyve, binds its substrate through this domain facilitating the turnover of $\mathrm{PI}(3) \mathrm{P}$ to $\mathrm{PI}(3,5) \mathrm{P}_{2}$ on maturing endocytic organelles (Ikonomov, Sbrissa et al., 2006; Rutherford, Traer et al., 2006; Shisheva, 2008).

The PX domain is a $\sim 130$ residue motif named after the two phagocyte NADPH oxidase (phox) subunits in which it was first described (Ponting, 1996). Whilst PI(3)P appears to be the preferred target of most PX domain containing proteins (Seet and Hong, 2006), a variety of other specificities have been reported. Sorting Nexins (SNX) 9 and 18 both bind PI(4,5) $\mathrm{P}_{2}$ where they appear to function in endocytic trafficking events at the plasma membrane and AP1-positive endosomal membranes respectively, whilst SNX1 is reported to bind both $\mathrm{PI}(3) \mathrm{P}$ and $\mathrm{PI}(3,5)_{2}$ and is involved in retrograde trafficking events from early endosomes to the Golgi (Carlton, Bujny et al., 2004; Haberg, Lundmark et al., 2008; Shin, Ahn et al., 2008; Soulet, Yarar et al., 2005; Yarar, Waterman-Storer et al., 2007). Interestingly, the PX domain in isolation is often not sufficient to recruit these molecules to PI-enriched membranes. 
Removal of the membrane curvature sensing and bending Bin Amphiphysin Rvs (BAR) from sorting nexin 1 (SNX1) renders it cytosolic demonstrating the necessity for the coincident detection of both the specific PIs and the appropriately curved membranes for recruitment to endosomes (Carlton, Bujny et al., 2005).

\section{Phosphoinositides in Salmonella Infection}

Early in the Salmonella invasion process cellular PI levels are reported to undergo rapid and dynamic shifts. HPLC analysis of lipids extracted from Salmonella-infected cells revealed remarkable elevation in the relative amounts of $\mathrm{PI}(4,5) \mathrm{P}_{2}, \mathrm{PI}(3,4) \mathrm{P}_{2}$ and $\mathrm{PI}(3,4,5) \mathrm{P}_{3}$ as well as more moderate elevation in PI(3)P when compared to control cells (Bakowski, Braun et al., 2010). Modulation of $\mathrm{PI}(4,5) \mathrm{P}_{2}$ and $\mathrm{PI}(3,4,5) \mathrm{P}_{2}$ serves to destabilise the cortical cytoskeleton leading to membrane ruffling, macropinosome formation and promoting bacterial uptake whilst the accumulation of $\mathrm{PI}(3) \mathrm{P}$ on membrane ruffles and the nascent SCV promotes recruitment of the PI(3)P-binding r-SNARE VAMP8 which facilitates fusion events within the endosomal network (Dai, Zhang et al., 2007).

Shortly after formation, the enveloping macropinosome shrinks to form an adherent SCV around one or more Salmonella. This process is mediated, at least in part, by a suite of PIbinding effector molecules known as the sorting nexins (SNX). The SNXs represent a collection of approximately 50 human proteins with diverse domain architectures that are recruited to intracellular membranes via a common PX domain (Cullen, 2008). It was revealed that SNXs 1 and 5 form heterodimers upon the surface of maturing PI(3)P-rich macropinosomes via a conserved BAR domain within their carboxyl-terminus. This dimerisation leads to the formation of tubular carriers that retrieve large quantities of membrane from and the consequent condensation of the macropinosome as the organelle matures (Kerr, Lindsay et al., 2006). The transient nature of the SNX's recruitment to the SCV was demonstrated to reflect the subsequent phosphorylation of $\mathrm{PI}(3) \mathrm{P}$ within the membrane of the macropinosome to $\mathrm{PI}(3,5) \mathrm{P}_{2}$ by the phosphatidylinositol 5-kinase, PIKfyve. This final PI transition is necessary for the ultimate fusion of macropinosomes with the late endosomal/lysosomal system (Kerr, Wang et al., 2010).

While the SCV appears to share properties in common with constitutive macropinosomes, including SNX-recruitment, the maturation process is significantly perturbed so that the SCV persists for hours. Hernandez et al. (2004) observed that unlike constitutive macropinosomes, SCVs containing wild-type Salmonella rapidly accumulate and maintain elevated levels of PI(3)P for up to 90mins (Hernandez, Hueffer et al., 2004). Bujney et al. demonstrated that one consequence of this was the elevated and sustained accumulation of SNX1 to the newly formed SCV. This in turn results in a grossly exaggerated tubulation event that leads to the rapid contraction of the enveloping SCV to form a tightly-wrapped adherent membrane around the bacterium (Bujny, Ewels et al., 2008). Time-lapse videomicroscopy elegantly demonstrates the precision with which the pathogen is able to manipulate its local environment as the tubulation and condensation of the SCV is not observed on the surrounding constitutive macropinosomes (see Figure 2). One might speculate that this embellished contraction may facilitate the eventual engagement of the SPI2-T3SS with the membrane of the SCV. 

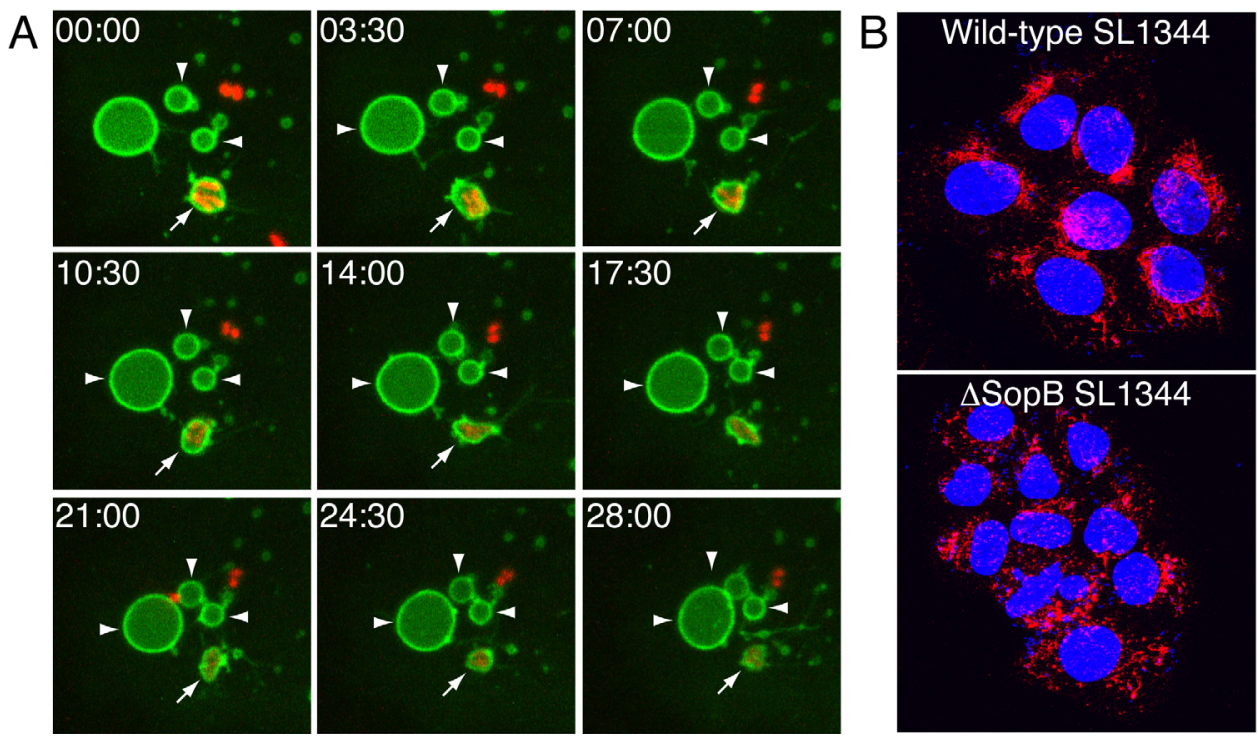

(A) A-431 cells transiently transfected with a mammalian expression construct encoding the highaffinity PI(3)P-probe 2xFYVEHrs fused with eGFP were infected with late-log phase wild-type Salmonella expressing RFP (RFP-SL1344) and imaged live using a Zeiss LSM 510 confocal scanning microscope. Initially spacious, the PI(3)P-rich SCV (arrows) is observed to undergo significant tubulation and in doing so condenses to form an adherent membrane around the bacteria (red). In contrast, surrounding constitutive macropinosomes (arrow heads) present limited tubulation and no condensation in the same time period. (B) This tubulation is mediated by SopB as evidenced by the more moderate tubulation observed in $\triangle \mathrm{SopB}$ strain-infected cells when compared to those infected with wild-type Salmonella.

Fig. 2. SNX-mediated tubulation leads to dramatic condensation of the SCV.

\subsection{SopB, a pleitropic phosphoinositide phosphatase}

This striking impact upon PI metabolism and SNX recruitment is achieved, at least in part, by directly modulating the PI-composition of the SCV through the delivery of virulence factors with PI-phosphatase activity. SopB (also called SigD) is a SPI-T3SS phosphoinositide phosphatase that has diverse influence upon the pathogenesis of S. typhimurium. It contributes to membrane sealing at the plasma membrane and actin-rearrangement through activation of SGEF (a guanine nucleotide exchange factor for RhoG), during bacterial invasion (Patel and Galan, 2006; Terebiznik, Vieira et al., 2002). It also inhibits induction of apoptosis through activation of Akt and promotes the early recruitment of Rab5 and its effector Vps34 to the SCV (Knodler, Finlay et al., 2005; Mallo, Espina et al., 2008; Steele-Mortimer, Knodler et al., 2000).

Sharing similarity with mammalian PI 4- and 5-phosphatases, SopB is reported to hydrolyse a variety of PIs in vitro, including $\mathrm{PI}(3,4) \mathrm{P}_{2}, \mathrm{PI}(3,5) \mathrm{P}_{2}$ and $\mathrm{PI}, 4,5 \mathrm{P}_{2}$, and more recently, $\mathrm{PI}(4,5) \mathrm{P}_{2}$ in vivo (Bakowski, Braun et al., 2010; Marcus, Wenk et al., 2001; Norris, Wilson et al., 1998). Given this apparent broad specificity and the capacity to promote Vps34 recruitment to, and presumably therefore de novo synthesis of PI(3)P on, the SCV, the wide ranging impact SopB has upon the infectious cycle of Salmonella is perhaps not surprising. Indeed, SopB was recently demonstrated to have profound affects upon the biophysical 
properties of the SCV itself. Bakowski et al. (2010) The authors used an mRFP-tagged derivative of the K-ras tail with all the serine and threonine residues mutated to alanine and the lysine residues mutated to arginine so that its recruitment to intracellular membranes was only governed by surface potential and not phosphorylation or ubiquitination. They demonstrated that by reducing the levels of negatively-charged lipids like $\mathrm{PI}(4,5) \mathrm{P}_{2}$ and phosphatidylserine on the nascent SCV, SopB activity orchestrates the dissociation of a number of endocytic Rab proteins and inhibits fusion of the SCV with bactericidal lysosomal compartments (Bakowski, Braun et al., 2010).

In addition to its lipid phosphatase activity, SopB was also recently revealed to interact with CDC42 suggesting an additional aspect to its contribution to the infectious process. Rodríguez-Escudero et al. (2011) demonstrated that a catalytically inactive SopB mutant can inhibit CDC42 but not Rac1 in a yeast model system. This interaction occurs independent of the activation state of CDC42. Interestingly, Salmonella strains harbouring SopB-mutations that render it unable to bind CDC42 presented similar invasion efficiencies when compared to the wild-type but reduced intracellular replication (Rodriguez-Escudero, Ferrer et al., 2011; Rodriguez-Escudero, Rotger et al., 2006). This indicates that the SopB-CDC42 association is pertinent to the intracellular adaptation of the pathogen rather than the actinremodeling that occurs upon invasion as one might expect.

\subsection{The sorting nexins coordinate Salmonella pathogenesis}

By manipulating cellular PI metabolism, Salmonella effectively exaggerates and alters the function of the PI-effector molecules thereby creating a niche within the cell that supports replication and infection. In the context of SNX1, this is manifest in SopB-dependent overrecruitment and tubulation events leading to the accelerated condensation of the organelle and formation of the adherent SCV (See Figure 2). siRNA-mediated suppression of SNX1 is sufficient to inhibit SCV progression into the cell and had a moderate impact upon the intracellular replication of Salmonella (Bujny, Ewels et al., 2008). Similarly, knockdown of SNX3, which constitutes little more than a PX domain and is found on tubular extensions of the SCV distinct from those of SNX1, inhibits SCV maturation and intracellular replication of Salmonella (Braun, Wong et al., 2010). But what of the other PX proteins?

Wang et al. (2010) employed an ectopic screening strategy to demonstrate that a specific cohort of the SNXs, namely SNXs 1, 5, 9, 18 and 33, could significantly elevate the rate of macropinocytosis in cultured monolayers suggesting specific roles beyond the tubulation events described earlier (Wang, Kerr et al., 2010). Perhaps these SNXs have unique roles to play in Salmonella pathogenicity? Towards this we have initiated a detailed examination of the recruitment of the SNXs to the nascent SCV. Immunofluorescent-labelling of infected cells transiently transfected with mammalian expression constructs encoding epitopetagged SNXs and co-labelled with endogenous SNX1 revealed 19 SNXs recruited to the SCV within the first $30 \mathrm{mins}$ of infection (see Figure 3).

Interestingly, significant variation in the precise distribution of these SNXs on the early SCV was observed. Those most related to SNX1, and those previous demonstrated to have a role in endosomal trafficking, namely SNXs 2, 4, 5, 6, 7 and 8, were found on the aforementioned SNX1-labelled tubular extensions of the SCV. mPLD2, SNX12, SNX16, SNX21, SNX23 and SNX28 were found evenly associated with the tubules and the body of the SCV 
proper whilst hPLD1, SNX10, SNX11, SNX15, SNX27 and p40phox were restricted to the body of the SCV only. The notable absence of SNX3 on the SCV perhaps reflects a difference in the temporal nature of the SNXs recruitment. Unlike SNX1, which is most evident on the SCV very early in the infection, SNX3 is recruited $\sim 60 \mathrm{mins}$ into the infection indicating additional levels of complexity in the mechanism of recruitment within this PI-effector family (Braun, Wong et al., 2010). Indeed the PX-containing subunits of the NADPH oxidase complex represent a significant threat to intracellular Salmonella, providing the means to deliver a bactericidal oxidative burst to the SCV in macrophages. Virulent Salmonella strains avoid this through a SPI2-dependent mechanism that inhibits trafficking or targeting of NADPH oxidase-containing vesicles to the vicinity of the SCV (Vazquez-Torres, $\mathrm{Xu}$ et al., 2000). It will be interesting to see the likely diverse roles played by the SNXs during Salmonella pathogenicity emerge in the near future.
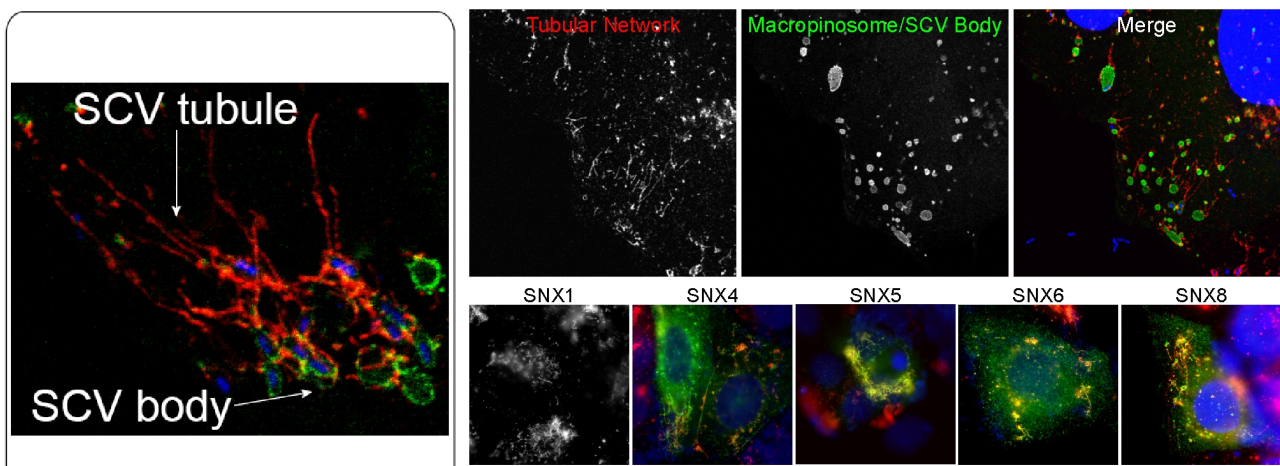

SNX5

SNX6 SNX8
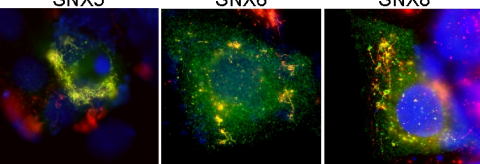

Localised to the SCV Localised to the SCV tubule only body only SNX1 SNX2 $\quad$ SNX10

SNX4 SNX11

SNX5 SNX15

SNX6 SNX27

SNX7 p40phox

SNX8

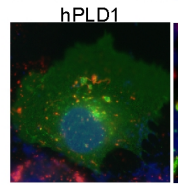

SNX15

SNX27

Localised to the SCV tubule \& body MPLD2

SNX12

SNX16

SNX21

SNX23 (KIF16B)

SNX28

\begin{tabular}{|l|}
\hline Not localised to the \\
SCV \\
SNX3 \\
SNX14 \\
SNX17 \\
SNX22 \\
SNX30 \\
hypthPX \\
p47phox \\
p170 \\
Sh3PXn2B
\end{tabular}
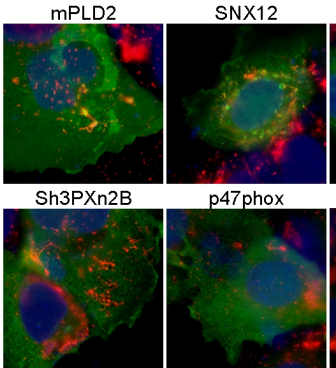

SNX12

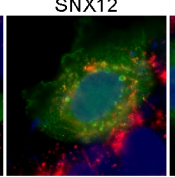

p40phox

SNX11
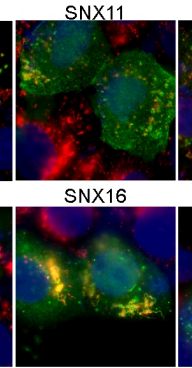

p170

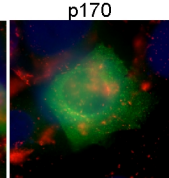

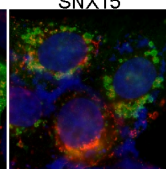

SNX21

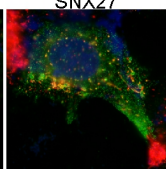

SNX28

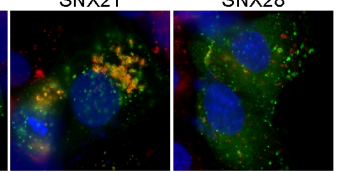

SNX22

SNX30
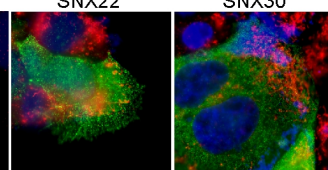

Fig. 3. Salmonella specifically and directly manipulates SNX recruitment to the nascent SCV. A431 cells transiently transfected with mammalian expression constructs encoding myc-epitope tagged SNXs were infected with late-log phase Salmonella (SL1344) for 30mins, fixed with 4\% PFA and labelled with an $\alpha$-myc polyclonal antibody, a monoclonal antibody specific for SNX1, DAPI and appropriate secondary antibodies. Images were captured using a Zeiss LSM 510 confocal scanning microscope. Images were examined and the recruitment of the overexpressed SNX construct (green) was compared with that of endogenous SNX1 (red). 


\subsection{Targeting the phosphoinositides for therapeutic intervention}

With the development of antibiotic resistance, new strategies to combat intracellular pathogens, like Salmonella, need to be developed. One emergent approach is to manipulate non-essential host cellular pathways required by the pathogen during its infectious cycle (Schwegmann and Brombacher, 2008). Given the frequent connections between the PIs, their effectors, and those of Salmonella, this protein-lipid network represents a potential opportunity for novel modes of intervention.

We recently demonstrated that disruption of $\mathrm{PI}(3,5) \mathrm{P}_{2}$-synthesis through perturbation of PIKfyve activity, be it by interfering mutant, siRNA-mediated knockdown or pharmacological means (YM201636), was led to a profound inhibition in the fusion of maturing macropinosomes with the late endosomal/lysosomal network. As mentioned earlier, these directed fusion events are necessary for the formation of SIFs during an infection with S. typhimurium. Remarkably, inhibition of PIKfyve was sufficient to halt SIF formation, SCV acidification, induction of the SPI2 Operon and ultimately intracellular replication of Salmonella whilst still maintaining the pathogen within an intracellular compartment (Kerr, Wang et al., 2010).

Of course a thorough understanding of the potential consequence of targeting PIs is required for such host-directed therapeutics to be effective. Inhibition of PI(3)P accumulation on the SCV with wortmannin is sufficient to halt recruitment of FYVE-domain containing molecules like EEA1 and even the SIF-marker LAMP1 but does not perturb intracellular replication of Salmonella (Scott, Cuellar-Mata et al., 2002). In fact, some have observed elevated rates of replication in host treated with wortmannin (Brumell, Tang et al., 2002). This is because, unlike those cultured in the presence of YM201626, the integrity of the SCV itself is disrupted releasing the bacteria into the cytosol, where they may freely replicate.

Aside from counteracting the evolution of bacterial antibiotic resistance, these host-directed therapeutics may provide broad-spectrum solutions to a variety of pathogens. Indeed, Salmonella is not unique in its partiality for targeting the PIs and associated molecules. Yersinia species activate $\mathrm{PI}(5)$ kinase to stimulate $\operatorname{PtdIns}(4,5) \mathrm{P}_{2}$ production at sites of bacterial invasion whilst Listeria monocytogenes and uropathogenic E. coli stimulate class I PI(3) kinase generating PtdIns(3,4,5) $\mathrm{P}_{3}$ to promote uptake (Gavicherla, Ritchey et al., 2010; Ireton, 2007; Ireton, Payrastre et al., 1999; Martinez, Mulvey et al., 2000). Mycobacterium tuberculosis inhibits class III PI(3) kinase, Vps34, involved in the formation of PI(3)P effectively arresting phagosome maturation (Chua and Deretic, 2004). Thus by targeting the PIs, we may be able to specifically interfere with a variety of infections at different stages of there pathogenic cycle.

\section{Conclusion}

Despite comprising less than $1 \%$ of cellular lipids, the PIs and their effectors are key regulators of intra- and inter-cellular signalling, cell growth and survival, cytoskeletal dynamics and membrane trafficking pathways. It is perhaps not surprising that intracellular pathogens that exploit these processes directly target this remarkably complex protein-lipid network during the infectious process. Whilst the roles the PIs play directly and through their effectors like the sorting nexins during a Salmonella infection are only now coming to light, there is already strong evidence to consider them as viable therapeutic targets for intervention. 


\section{Acknowledgments}

This work was supported by funding from the National Health and Medical Research Council (NHMRC) of Australia (606788). RDT is supported by NHMRC Senior Research Fellowship (511042). Microscopy was performed at the Australian Cancer Research Foundation (ACRF)/Institute for Molecular Bioscience Dynamic Imaging Facility for Cancer Biology, which was established with the support of the ACRF.

\section{References}

Bakowski, M.A., Braun, V., Lam, G.Y., Yeung, T., Heo, W.D., Meyer, T., Finlay, B.B., Grinstein, S., \& Brumell, J.H. (2010). The phosphoinositide phosphatase SopB manipulates membrane surface charge and trafficking of the Salmonella-containing vacuole. Cell Host Microbe 7, 453-462.

Braun, V., Wong, A., Landekic, M., Hong, W.J., Grinstein, S., \& Brumell, J.H. (2010). Sorting nexin 3 (SNX3) is a component of a tubular endosomal network induced by Salmonella and involved in maturation of the Salmonella-containing vacuole. Cell Microbiol 12, 1352-1367.

Brumell, J.H., Tang, P., Zaharik, M.L., \& Finlay, B.B. (2002). Disruption of the Salmonellacontaining vacuole leads to increased replication of Salmonella enterica serovar typhimurium in the cytosol of epithelial cells. Infect Immun 70, 3264-3270.

Bujny, M.V., Ewels, P.A., Humphrey, S., Attar, N., Jepson, M.A., \& Cullen, P.J. (2008). Sorting nexin-1 defines an early phase of Salmonella-containing vacuoleremodeling during Salmonella infection. J Cell Sci 121, 2027-2036.

Carlton, J., Bujny, M., Peter, B.J., Oorschot, V.M., Rutherford, A., Mellor, H., Klumperman, J., McMahon, H.T., \& Cullen, P.J. (2004). Sorting nexin-1 mediates tubular endosometo-TGN transport through coincidence sensing of high- curvature membranes and 3-phosphoinositides. Curr Biol 14, 1791-1800.

Carlton, J., Bujny, M., Rutherford, A., \& Cullen, P. (2005). Sorting nexins--unifying trends and new perspectives. Traffic 6, 75-82.

Chua, J., \& Deretic, V. (2004). Mycobacterium tuberculosis reprograms waves of phosphatidylinositol 3-phosphate on phagosomal organelles. J Biol Chem 279, 36982-36992.

Cooke, F.T. (2002). Phosphatidylinositol 3,5-bisphosphate: metabolism and function. Arch Biochem Biophys 407, 143-151.

Cooke, F.T., Dove, S.K., McEwen, R.K., Painter, G., Holmes, A.B., Hall, M.N., Michell, R.H., \& Parker, P.J. (1998). The stress-activated phosphatidylinositol 3-phosphate 5kinase Fab1p is essential for vacuole function in S. cerevisiae. Curr Biol 8, 12191222.

Cronin, T.C., DiNitto, J.P., Czech, M.P., \& Lambright, D.G. (2004). Structural determinants of phosphoinositide selectivity in splice variants of Grp1 family PH domains. Embo J 23, 3711-3720.

Cullen, P.J. (2008). Endosomal sorting and signalling: an emerging role for sorting nexins. Nat Rev Mol Cell Biol 9, 574-582.

Dai, S., Zhang, Y., Weimbs, T., Yaffe, M.B., \& Zhou, D. (2007). Bacteria-generated PtdIns(3)P recruits VAMP8 to facilitate phagocytosis. Traffic 8, 1365-1374. 
Di Paolo, G., \& De Camilli, P. (2006). Phosphoinositides in cell regulation and membrane dynamics. Nature 443, 651-657.

DiNitto, J.P., \& Lambright, D.G. (2006). Membrane and juxtamembrane targeting by PH and PTB domains. Biochim Biophys Acta 1761, 850-867.

Dove, S.K., McEwen, R.K., Cooke, F.T., Parker, P.J., \& Michell, R.H. (1999). Phosphatidylinositol 3,5-bisphosphate: a novel lipid that links stress responses to membrane trafficking events. Biochem Soc Trans 27, 674-677.

Dove, S.K., McEwen, R.K., Mayes, A., Hughes, D.C., Beggs, J.D., \& Michell, R.H. (2002). Vac14 controls PtdIns $(3,5) \mathrm{P}(2)$ synthesis and Fab1-dependent protein trafficking to the multivesicular body. Curr Biol 12, 885-893.

Dove, S.K., Piper, R.C., McEwen, R.K., Yu, J.W., King, M.C., Hughes, D.C., Thuring, J., Holmes, A.B., Cooke, F.T., Michell, R.H., Parker, P.J., \& Lemmon, M.A. (2004). Svp1p defines a family of phosphatidylinositol 3,5-bisphosphate effectors. Embo J 23, 1922-1933.

Garcia-del Portillo, F., \& Finlay, B.B. (1995). Targeting of Salmonella typhimurium to vesicles containing lysosomal membrane glycoproteins bypasses compartments with mannose 6-phosphate receptors. J Cell Biol 129, 81-97.

Garcia-del Portillo, F., Zwick, M.B., Leung, K.Y., \& Finlay, B.B. (1993a). Intracellular replication of Salmonella within epithelial cells is associated with filamentous structures containing lysosomal membrane glycoproteins. Infect Agents Dis 2, 227231.

Garcia-del Portillo, F., Zwick, M.B., Leung, K.Y., \& Finlay, B.B. (1993b). Salmonella induces the formation of filamentous structures containing lysosomal membrane glycoproteins in epithelial cells. Proc Natl Acad Sci U S A 90, 10544-10548.

Gary, J.D., Wurmser, A.E., Bonangelino, C.J., Weisman, L.S., \& Emr, S.D. (1998). Fab1p is essential for PtdIns(3)P 5-kinase activity and the maintenance of vacuolar size and membrane homeostasis. J Cell Biol 143, 65-79.

Gavicherla, B., Ritchey, L., Gianfelice, A., Kolokoltsov, A.A., Davey, R.A., \& Ireton, K. (2010). Critical role for the host GTPase-activating protein ARAP2 in InlB-mediated entry of Listeria monocytogenes. Infect Immun 78, 4532-4541.

Haberg, K., Lundmark, R., \& Carlsson, S.R. (2008). SNX18 is an SNX9 paralog that acts as a membrane tubulator in AP-1-positive endosomal trafficking. J Cell Sci 121, 14951505.

Haigler, H.T., McKanna, J.A., \& Cohen, S. (1979). Rapid stimulation of pinocytosis in human carcinoma cells A-431 by epidermal growth factor. J Cell Biol 83, 82-90.

Haraga, A., Ohlson, M.B., \& Miller, S.I. (2008). Salmonellae interplay with host cells. Nat Rev Microbiol 6, 53-66.

Hernandez, L.D., Hueffer, K., Wenk, M.R., \& Galan, J.E. (2004). Salmonella modulates vesicular traffic by altering phosphoinositide metabolism. Science 304, 1805-1807.

Ikonomov, O.C., Sbrissa, D., \& Shisheva, A. (2006). Localized PtdIns 3,5-P2 synthesis to regulate early endosome dynamics and fusion. Am J Physiol Cell Physiol 291, C393-404.

Ireton, K. (2007). Entry of the bacterial pathogen Listeria monocytogenes into mammalian cells. Cell Microbiol 9, 1365-1375.

Ireton, K., Payrastre, B., \& Cossart, P. (1999). The Listeria monocytogenes protein InlB is an agonist of mammalian phosphoinositide 3-kinase. J Biol Chem 274, 17025-17032. 
Kerr, M.C., Lindsay, M.R., Luetterforst, R., Hamilton, N., Simpson, F., Parton, R.G., Gleeson, P.A., \& Teasdale, R.D. (2006). Visualisation of macropinosome maturation by the recruitment of sorting nexins. J Cell Sci 119, 3967-3980.

Kerr, M.C., \& Teasdale, R.D. (2009). Defining macropinocytosis. Traffic 10, 364-371.

Kerr, M.C., Wang, J.T., Castro, N.A., Hamilton, N.A., Town, L., Brown, D.L., Meunier, F.A., Brown, N.F., Stow, J.L., \& Teasdale, R.D. (2010). Inhibition of the PtdIns(5) kinase PIKfyve disrupts intracellular replication of Salmonella. Embo J 29, 1331-1347.

Knodler, L.A., Finlay, B.B., \& Steele-Mortimer, O. (2005). The Salmonella effector protein SopB protects epithelial cells from apoptosis by sustained activation of Akt. J Biol Chem 280, 9058-9064.

Krauss, M., \& Haucke, V. (2007). Phosphoinositide-metabolizing enzymes at the interface between membrane traffic and cell signalling. EMBO Rep 8, 241-246.

Lemmon, M.A., \& Ferguson, K.M. (2000). Signal-dependent membrane targeting by pleckstrin homology (PH) domains. Biochem J 350 Pt 1, 1-18.

Levine, T.P., \& Munro, S. (2002). Targeting of Golgi-specific pleckstrin homology domains involves both PtdIns 4-kinase-dependent and -independent components. Curr Biol 12, 695-704.

Mallo, G.V., Espina, M., Smith, A.C., Terebiznik, M.R., Aleman, A., Finlay, B.B., Rameh, L.E., Grinstein, S., \& Brumell, J.H. (2008). SopB promotes phosphatidylinositol 3phosphate formation on Salmonella vacuoles by recruiting Rab5 and Vps34. J Cell Biol 182, 741-752.

Marcus, S.L., Wenk, M.R., Steele-Mortimer, O., \& Finlay, B.B. (2001). A synaptojaninhomologous region of Salmonella typhimurium SigD is essential for inositol phosphatase activity and Akt activation. FEBS Lett 494, 201-207.

Martinez, J.J., Mulvey, M.A., Schilling, J.D., Pinkner, J.S., \& Hultgren, S.J. (2000). Type 1 pilus-mediated bacterial invasion of bladder epithelial cells. Embo J 19, 2803-2812.

Norris, F.A., Wilson, M.P., Wallis, T.S., Galyov, E.E., \& Majerus, P.W. (1998). SopB, a protein required for virulence of Salmonella dublin, is an inositol phosphate phosphatase. Proc Natl Acad Sci U S A 95, 14057-14059.

Odorizzi, G., Babst, M., \& Emr, S.D. (1998). Fab1p PtdIns(3)P 5-kinase function essential for protein sorting in the multivesicular body. Cell 95, 847-858.

Panaretou, C., Domin, J., Cockcroft, S., \& Waterfield, M.D. (1997). Characterization of p150, an adaptor protein for the human phosphatidylinositol (PtdIns) 3-kinase. Substrate presentation by phosphatidylinositol transfer protein to the p150.Ptdins 3-kinase complex. J Biol Chem 272, 2477-2485.

Patel, J.C., \& Galan, J.E. (2006). Differential activation and function of Rho GTPases during Salmonella-host cell interactions. J Cell Biol 175, 453-463.

Ponting, C.P. (1996). Novel domains in NADPH oxidase subunits, sorting nexins, and PtdIns 3-kinases: binding partners of SH3 domains? Protein Sci 5, 2353-2357.

Rodriguez-Escudero, I., Ferrer, N.L., Rotger, R., Cid, V.J., \& Molina, M. (2011). Interaction of the Salmonella Typhimurium effector protein SopB with host cell Cdc42 is involved in intracellular replication. Mol Microbiol 80, 1220-1240.

Rodriguez-Escudero, I., Rotger, R., Cid, V.J., \& Molina, M. (2006). Inhibition of Cdc42dependent signalling in Saccharomyces cerevisiae by phosphatase-dead SigD/SopB from Salmonella typhimurium. Microbiology 152, 3437-3452. 
Rutherford, A.C., Traer, C., Wassmer, T., Pattni, K., Bujny, M.V., Carlton, J.G., Stenmark, H., \& Cullen, P.J. (2006). The mammalian phosphatidylinositol 3-phosphate 5-kinase (PIKfyve) regulates endosome-to-TGN retrograde transport. J Cell Sci 119, 39443957.

Schwegmann, A., \& Brombacher, F. (2008). Host-directed drug targeting of factors hijacked by pathogens. Sci Signal 1, re8.

Scott, C.C., Cuellar-Mata, P., Matsuo, T., Davidson, H.W., \& Grinstein, S. (2002). Role of 3phosphoinositides in the maturation of Salmonella-containing vacuoles within host cells. J Biol Chem 277, 12770-12776.

Seet, L.F., \& Hong, W. (2006). The Phox (PX) domain proteins and membrane traffic. Biochim Biophys Acta 1761, 878-896.

Shin, N., Ahn, N., Chang-Ileto, B., Park, J., Takei, K., Ahn, S.G., Kim, S.A., Di Paolo, G., \& Chang, S. (2008). SNX9 regulates tubular invagination of the plasma membrane through interaction with actin cytoskeleton and dynamin 2. J Cell Sci 121, 12521263.

Shisheva, A. (2008). PIKfyve: Partners, significance, debates and paradoxes. Cell Biol Int 32, 591-604.

Soulet, F., Yarar, D., Leonard, M., \& Schmid, S.L. (2005). SNX9 regulates dynamin assembly and is required for efficient clathrin-mediated endocytosis. Mol Biol Cell 16, 20582067.

Steele-Mortimer, O., Knodler, L.A., Marcus, S.L., Scheid, M.P., Goh, B., Pfeifer, C.G., Duronio, V., \& Finlay, B.B. (2000). Activation of Akt/protein kinase B in epithelial cells by the Salmonella typhimurium effector sigD. J Biol Chem 275, 37718-37724.

Swanson, J.A., \& Watts, C. (1995). Macropinocytosis. Trends Cell Biol 5, 424-428.

Terebiznik, M.R., Vieira, O.V., Marcus, S.L., Slade, A., Yip, C.M., Trimble, W.S., Meyer, T., Finlay, B.B., \& Grinstein, S. (2002). Elimination of host cell PtdIns $(4,5) P(2)$ by bacterial SigD promotes membrane fission during invasion by Salmonella. Nat Cell Biol 4, 766-773.

Vanhaesebroeck, B., Leevers, S.J., Ahmadi, K., Timms, J., Katso, R., Driscoll, P.C., Woscholski, R., Parker, P.J., \& Waterfield, M.D. (2001). Synthesis and function of 3phosphorylated inositol lipids. Annu Rev Biochem 70, 535-602.

Vazquez-Torres, A., Xu, Y., Jones-Carson, J., Holden, D.W., Lucia, S.M., Dinauer, M.C., Mastroeni, P., \& Fang, F.C. (2000). Salmonella pathogenicity island 2-dependent evasion of the phagocyte NADPH oxidase. Science 287, 1655-1658.

Vicinanza, M., D'Angelo, G., Di Campli, A., \& De Matteis, M.A. (2008). Function and dysfunction of the PI system in membrane trafficking. Embo J 27, 2457-2470.

Wang, J.T., Kerr, M.C., Karunaratne, S., Jeanes, A., Yap, A.S., \& Teasdale, R.D. (2010). The SNX-PX-BAR family in macropinocytosis: the regulation of macropinosome formation by SNX-PX-BAR proteins. PLoS One 5, e13763.

Wang, Y.J., Wang, J., Sun, H.Q., Martinez, M., Sun, Y.X., Macia, E., Kirchhausen, T., Albanesi, J.P., Roth, M.G., \& Yin, H.L. (2003). Phosphatidylinositol 4 phosphate regulates targeting of clathrin adaptor AP-1 complexes to the Golgi. Cell 114, 299310. 
Yamamoto, A., DeWald, D.B., Boronenkov, I.V., Anderson, R.A., Emr, S.D., \& Koshland, D. (1995). Novel PI(4)P 5-kinase homologue, Fab1p, essential for normal vacuole function and morphology in yeast. Mol Biol Cell 6, 525-539.

Yarar, D., Waterman-Storer, C.M., \& Schmid, S.L. (2007). SNX9 couples actin assembly to phosphoinositide signals and is required for membrane remodeling during endocytosis. Dev Cell 13, 43-56.

Zerial, M., \& McBride, H. (2001). Rab proteins as membrane organizers. Nat Rev Mol Cell Biol 2, 107-117. 
(C) 2012 The Author(s). Licensee IntechOpen. This is an open access article distributed under the terms of the Creative Commons Attribution 3.0 License, which permits unrestricted use, distribution, and reproduction in any medium, provided the original work is properly cited. 